\title{
Aplicación de un material vitrocerámico a la biorremediación de metales pesados
}

\author{
A. M. GARCÍA', J. M. VILLORA², D. A. MORENO', C. RANNINGER', P. CALLEJAS², M. F. BARBA² \\ ${ }^{1}$ Universidad Politécnica de Madrid, Dto de Ingeniería y Ciencia de los Materiales, Esc. Téc. Superior de Ingenieros Industriales, Madrid (España) \\ ${ }^{2}$ Instituto de Cerámica y Vidrio, CSIC, Kelsen s/n, Campus de Cantoblanco. Madrid (España)
}

\begin{abstract}
En este trabajo se elaboró un material vitrocerámico conteniendo la fase de hidroxiapatita, partiendo de residuos industriales y urbanos. Sobre la superficie del material se desarrollaron biopelículas de los microorganismos que aparecen habitualmente en las aguas residuales. Se hicieron ensayos de retención usando distintos metales pesados para evaluar la capacidad del material de eliminar tales elementos de un medio acuoso en presencia y ausencia de la biopelícula. Los resultados ponen de manifiesto la participación microbiana en los procesos de eliminación de los metales pesados del medio acuoso y ofrecen la posibilidad de utilizar el material vitrocerámico en un proceso de biorremediación de aguas contaminadas por metales pesados.
\end{abstract}

Palabras clave: Biorremediación, biopelícula, microorganismo, metal pesado, material vitrocerámico.

\section{Aplication of glassceramic material to the bioremediation of heavy metals}

In this study, a glassceramic material with hydroxyapatite phase was manufactured from industrial and urban wastes. On this material, biofilms from the microorganisms usually appearing in wastewater were developed. Batch assays were made using different heavy metals to analyze glassceramic material capacity to retain these elements from an aqueous medium in biofilm presence and absence. The results suggest that microorganisms are implicated in the removing of heavy metals from the aqueous medium and open the possibility to use the glassceramic material in a bioremediation process.

Keywords: Bioremediation, biofilm, microorganism, heavy metal, glassceramic material.

\section{INTRODUCCIÓN}

Durantelosúltimos años, la toxicidad y el potencial bioacumulativo de los metales pesados han desencadenado gran interés por desarrollar métodos que faciliten su eliminación del medio ambiente. La mayoría de los procesos químicos que se emplean actualmente para su tratamiento resultan muy caros y poco específicos $(1,2)$ por lo que se ha empezado a considerar la capacidad natural de muchos microorganismos de capturar y eliminar los metales pesados (3), proceso conocido como biorremediación. La mejor forma de explotar esta capacidad microbiana es inmovilizando los microorganismos en forma de biopelícula sobre un soporte adecuado en un biorreactor, lo que permite retenerlos con baja tasa de crecimiento y aumentar su concentración (4). El soporte ideal es aquel con gran superficie por unidad de volumen, barato, de alta durabilidad y que no se colapse fácilmente. Los materiales vitrocerámicos, aparte de reunir todas estas características, presentan una elevada estabilidad química y termodinámica que permite regenerarlos elevando la temperatura lo suficiente para quemar la materia biológica $(5,6)$, por lo que resultan una alternativa bastante atractiva en este sentido. Además, se ha comprobado su capacidad en la retención de metales pesados del agua $(7,8)$.

En este trabajo se desarrolló un material vitrocerámico del sistema $\mathrm{SiO}_{2}-\mathrm{P}_{2} \mathrm{O}_{5}-\mathrm{CaO}-\mathrm{Al}_{2} \mathrm{O}_{3}-\mathrm{X}_{2} \mathrm{O}$ (X: elemento alcalino) a partir de materias primas alternativas. Sobre su superficie se desarrollaron biopelículas de los microorganismos que aparecen en las aguas residuales. Se realizaron ensayos de retención de metales pesados usando distintas concentraciones de cadmio y plomo para evaluar la capacidad del material de retener dichos elementos de un medio acuoso en presencia y ausencia de la biopelícula microbiana.

\section{MATERIAL Y MÉTODOS}

\subsection{Material vitrocerámico}

Se elaboró un material vitrocerámico (Figura 1) dentro del sistema $\mathrm{SiO}_{2}-\mathrm{P}_{2} \mathrm{O}_{5}-\mathrm{CaO}-\mathrm{Al}_{2} \mathrm{O}_{3}-\mathrm{X}_{2} \mathrm{O}$ (X: elemento alcalino) a partir de residuos industriales y urbanos (casco de vidrio, harina de huesos de animales, tierra de diatomeas utilizadas y desechadas de la industria cervecera, lodos de la industria papelera, feldespatos y dolomitas que no cumplen especificaciones para cerámica blanca). Se prepararon probetas porosas de dimensiones $35 \times 15 \times 3 \mathrm{~mm}$ por el método de la esponja polimérica (9). La composición química del material es $14 \%$ de $\mathrm{P}_{2} \mathrm{O}_{5^{\prime}} 28 \%$ de $\mathrm{CaO}$, $50 \%$ de $\mathrm{SiO}_{2}$, $\%$ de óxidos alcalinos, $5 \%$ de $\mathrm{Al}_{2} \mathrm{O}_{3}$ y restos minoritarios de $\mathrm{MgO}$ y $\mathrm{Fe}_{2} \mathrm{O}_{3}$. Por difracción de rayos $\mathrm{X}$, se detectaron fases cristalinas de hidroxiapatita y en menor proporción otros fosfatos de calcio, tridimita, silicatos de calcio y fosfosilicatos de calcio, que se forman por reacción química durante el tratamiento térmico.

\subsection{Microorganismo}

El microorganismo empleado en este estudio se aisló de una muestra de agua residual tomada de la laguna de estabilización de la estación depuradora de La Poveda (Arganda del Rey, Madrid). Tras extraer su material genético y secuenciar el gen que codifica para el ADN ribosómico de la subunidad 16S (16S rDNA), se comparó con las secuencias depositadas en las bases de datos del EMBL (European Molecular Biology Laboratory) y NCBI (Nacional Center for Biotechnology Information). El microorganismo seleccionado resultó ser una bacteria entero-hemorrágica, Escherichia coli 0157:H7 (99\% de homología con el microorganismo con número de acceso AE005607). 


\subsection{Crecimiento de microorganismos en presencia de metales pesados}

Para conocer la capacidad de desarrollo de E. coli en presencia de metales pesados se estudiaron sus curvas de crecimiento a diferentes concentraciones de cadmio y de plomo (10 mg/L y $100 \mathrm{mg} / \mathrm{L}$ de cadmio $\mathrm{y}$ de plomo, por separado y conjuntamente) en medio líquido TSB (Tryptone Soya Broth, CM 129 Oxoid). Estos elementos se suministraron en forma de nitratos para evitar el empleo de ácido nítrico, que podría afectar al pH del medio. En todos los casos se preparó una solución patrón con una concentración de $1000 \mathrm{mg} / \mathrm{L}$, a partir de la cual se consiguieron las concentraciones de metales pesados deseadas, y se midió el pH de las soluciones comprobando que se encontraban en torno a un $\mathrm{pH}$ neutro. La concentración de metales pesados en el medio de cultivo se analizó por ICP-OES (espectrometría de emisión atómica con fuente con plasma de acoplamiento inductivo).

Los cultivos de E. coli en TSB se incubaron a $30^{\circ} \mathrm{C}$ y se tomaron muestras periódicamente para hacer diluciones seriadas en solución salina estéril ( $9 \mathrm{~g} / \mathrm{L} \mathrm{NaCl}$ en agua destilada) y siembra en placas de TSB (Tryptone Soya Agar, CM129 Oxoid supplemented with Agar (UPS) purissimen, Panreac) para recuento del número de unidades formadoras de colonia por $\mathrm{mL}(\mathrm{UFC} / \mathrm{mL})$.

\subsection{Desarrollo de biopelículas sobre el material vitrocerámico}

Con el fin de evaluar la capacidad del microorganismo seleccionado de desarrollarse sobre la superficie del material vitrocerámico y formar biopelículas se tomó un inóculo de $10^{6}$ células $/ \mathrm{mL}$, obtenido de un cultivo de $24 \mathrm{~h}$ en TSB, y se puso en contacto con la probeta paralepípeda de material vitrocerámico, previamente esterilizada en autoclave, en medio líquido TSB. Tras $24 \mathrm{~h}$ a $30^{\circ} \mathrm{C}$, se sacaron las probetas del medio de cultivo, se lavaron dos veces con agua destilada estéril para eliminar los microorganismos planctónicos no adheridos a la superficie del material y se prepararon las probetas para su estudio por Microscopía Electrónica de Barrido (SEM).

\subsection{Ensayos de retención de metales pesados}

Se colocaron probetas de material vitrocerámico, colonizadas durante $24 \mathrm{~h}$ por un cultivo estandarizado $\left(10^{6}\right.$ células $\left./ \mathrm{mL}\right)$ de $E$. coli, en medio líquido TSB con diferentes concentraciones de los metales pesados en estudio (10 mg/L Cd y $10 \mathrm{mg} / \mathrm{L} \mathrm{Pb}$ por separado y conjuntamente). Se incubaron a $30{ }^{\circ} \mathrm{C}$ durante $10 \mathrm{~h}$, tiempo estimado en el que se alcanza la fase estacionaria de crecimiento del microorganismo, agitándose suavemente cada hora para homogeneizar los metales pesados de la solución. Transcurrido este periodo, se sacaron las probetas del medio de cultivo, se lavaron dos veces con agua destilada estéril y se prepararon para su análisis por SEM. El medio de cultivo se esterilizó en autoclave para eliminar los posibles microorganismos planctónicos y se analizó por ICP-OES para determinar la concentración de metales pesados en el medio.

El mismo ensayo se realizó sin la adición del inóculo bacteriano para poder comparar la retención de metales pesados por el material en presencia y en ausencia de la biopelícula microbiana y determinar así la influencia de ésta en la inmovilización de los metales.

\subsection{Espectrometría de emisión atómica por fuente de plasma de acoplamiento inductivo (ICP-OES)}

La concentración de metales pesados de las diferentes soluciones se midió en un espectrómetro Thermo Jarrell Ash modelo IRIS ADVANTAGE con sistema de visualización axial y óptica auxiliar para una visión radial, con una fuente de radiofrecuencia de 40,68 $\mathrm{MHz}$ y red de difracción del

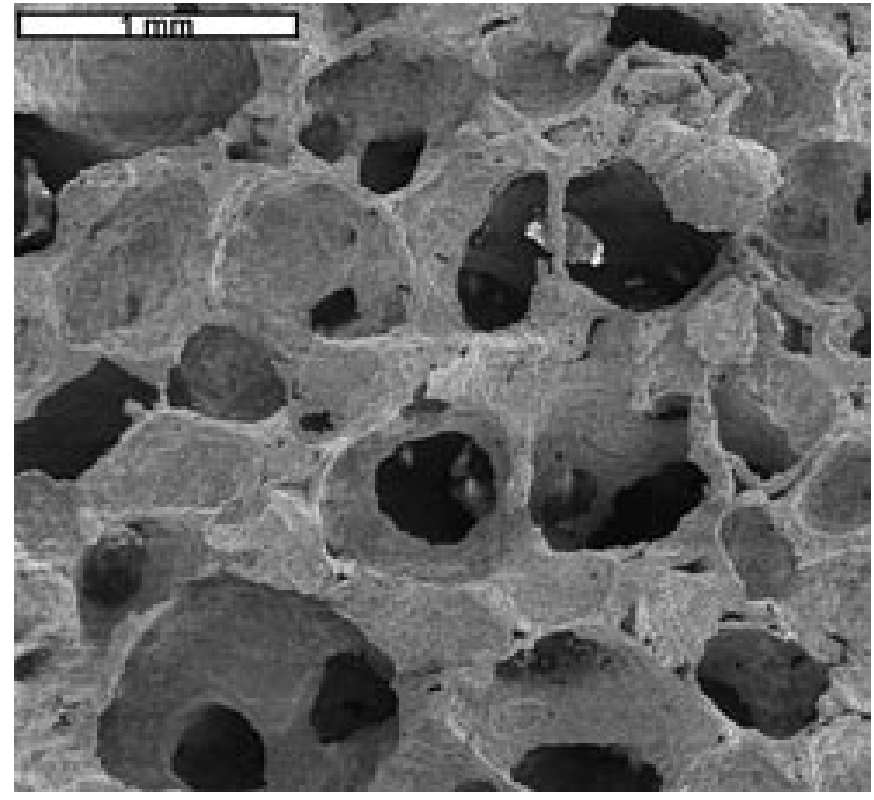

Figura 1. Estructura del material vitrocerámico por Microscopía Electrónica de Barrido (SEM).

tipo Echelle, dispersión cruzada por prisma y un mecanismo de inyección de carga (CID) como detector de estado sólido por lo que se pueden realizar análisis de varios elementos simultáneamente.

\subsection{Microscopía Electrónica de Barrido (SEM)}

Las probetas de material vitrocerámico de los diferentes ensayos, se fijaron en glutaraldehído al 2,5\% (V/V) en cacodilato sódico 0,01M durante 3 horas a $4{ }^{\circ} \mathrm{C}$. A continuación, se deshidrataron mediante inmersión en una serie acetónica creciente $(20 \%, 40 \%, 60 \%, 80 \%$ y $100 \%$ ) de 30 minutos cada una a $4{ }^{\circ} \mathrm{C}$. Posteriormente, se prepararon las probetas por punto crítico (Balzers Union CPD 020) y se metalizaron con oro (Balzers Union SCD 004) para ser observadas por SEM (Zeiss DSM 960) a $15 \mathrm{keV}$ en modo de electrones secundarios.

\section{RESULTADOS Y DISCUSIÓN}

\subsection{Crecimiento del microorganismo en presencia de metales pesados}

En la Figura 2 pueden observarse las curvas de crecimiento de $E$. coli en presencia de las distintas concentraciones de los metales pesados en estudio. Tanto a $10 \mathrm{mg} / \mathrm{L}$ como a $100 \mathrm{mg} / \mathrm{L}$ de cadmio y de plomo por separado y conjuntamente, muestra un crecimiento exponencial hasta aproximadamente las $8 \mathrm{~h}$ del cultivo, momento en el que alcanza la fase estacionaria de crecimiento que se mantiene durante las $30 \mathrm{~h}$ de duración del ensayo. Aunque a $100 \mathrm{mg} / \mathrm{L}$ se observa un desarrollo microbiano algo menor, no existe inhibición del crecimiento por la presencia de los metales pesados. Puede decirse que el microorganismo cuenta, por tanto, con la capacidad de resistir altas concentraciones de los citados metales, (concentraciones bastante más elevadas de las que normalmente aparecen en la depuradora de La Poveda) los cuales resultan tóxicos para otros microorganismos (10). Estudios previos (11) ponen de manifiesto que en la resistencia de $E$. coli a estos metales pesados se encuentran implicadas dos enzimas superoxido dismutasas (Fe-SOD y Mn-SOD) que protegen al microorganismo del estrés oxidativo provocado por los metales. Asimismo, Grimaud y cols. (12) han identificado una nueva enzima, metionina sulfóxido reductasa B (Msr B), que junto con la ya conocida MsrA contribuirían a la resistencia de E. coli al cadmio. 


\subsection{Desarrollo de biopelículas sobre el material vitrocerámico}

Cuando se analizó la capacidad de E. coli de formar biopelículas sobre el material vitrocerámico en estudio, se observó que el microorganismo se desarrolla perfectamente sobre la superficie de las probetas (Figura 3). A las 10 horas puede observarse por SEM colonias bacterianas adheridas a las irregularidades del material, que van uniéndose entre sí a través de sustancias poliméricas extracelulares (EPS) y a las $24 \mathrm{~h}$ se detectan auténticas biopelículas. Este es un mecanismo que permite a los microorganismos formar una matriz en la que quedan embebidos junto con otras sustancias, como nutrientes, y que les permite protegerse de factores externos adversos como por ejemplo la toxicidad producida por los metales pesados (13).

\subsection{Ensayos de retención de metales pesados}

El desarrollo de E. coli sobre la probeta de material vitrocerámico y en presencia de los metales pesados se observó por SEM. Este microorganismo hace que aumente notablemente la cantidad de metal pesado retenido sobre el material, de forma que el cadmio retenido pasa de un $6 \%$ cuando el material está estéril a un $98 \%$ cuando está colonizado por E. coli y el plomo de un $17 \%$ a un $39 \%$, respectivamente. Cuando ambos metales se encuentran simultáneamente en el medio de cultivo, se observa también un aumento considerable en la eliminación de metales del medio por los microorganismos, pero mientras que la cantidad de $\mathrm{Cd}$ retenida es similar a lo que ocurría cuando el elemento se encontraba en solitario (96\%), en el caso del $\mathrm{Pb}$ se retiene mucha mayor cantidad (58\%) cuando también está presente el Cd (Tabla 1), lo que pone de manifiesto las interacciones entre los iones metálicos. Low y cols. (14) en un estudio de biosorción de cadmio y plomo en una solución acuosa detectaron también esta mayor afinidad del plomo por unirse a los sitios de unión de la superficie bacteriana cuando ambos metales se encuentran juntos.

TABla I. ENSAYOS DE RETENCIÓN DE METALES. CONCENTRACIÓN DE METALES PESADOS, EN mg/L, MEDIDOS POR ICP-OES EN LAS DISTINTAS SOLUCIONES. CONTROL: SOLUCIÓN INICIAL, TRAS LA ADICIÓN DE $10 \mathrm{mg} / \mathrm{L}$ DEL METAL. MATERIAL: SOLUCIÓN TRAS EL ENSAYO CON EL MATERIAL VITROCERÁMICO EN AUSENCIA DE MICROORGANISMOS. BIOPELÍ́CULA: SOLUCIÓN TRAS EL ENSAYO CON EL MATERIAL VITROCERÁMICO PREVIAMENTE COLONIZADO POR UN CULTIVO DE 24 H DE E. COLI.

\begin{tabular}{|c|c|c|c|c|}
\hline Ensayo & Elemento & Control & Material & Biopelícula \\
\hline $10 \mathrm{mg} / \mathrm{L}$ Cadmio & $\mathrm{Cd}$ & 9,06 & 8,52 & 0,15 \\
\hline $10 \mathrm{mg} / \mathrm{L}$ Plomo & $\mathrm{Pb}$ & 9,69 & 7,99 & 5,92 \\
\hline $\begin{array}{c}10 \mathrm{mg} / \mathrm{L} \text { Cadmio }+10 \mathrm{mg} / \mathrm{L} \\
\text { Plomo }\end{array}$ & $\mathrm{Cd}$ & 9,32 & 8,61 & 0,36 \\
\cline { 2 - 5 } & $\mathrm{Pb}$ & 9,73 & 7,91 & 4,07 \\
\hline
\end{tabular}

\section{CONCLUSIONES}

El material vitrocerámico que se ha desarrollado en este trabajo constituye un filtro biológico ideal pues no sólo permite el desarrollo de los microorganismos sobre su superficie, sino que además es capaz de retener metales pesados por sí mismo. Conviene resaltar que se trata de un material desarrollado a partir de residuos industriales y urbanos por lo que contribuye al reciclado (casco de vidrio) y reducción de otros tipos de residuos (lodos de industrias papeleras, residuos de industria cervecera, etc.) además de los metales pesados.

Aparte de la retención de los metales pesados en el material vitrocerámico, se están produciendo fenómenos de inmovilización
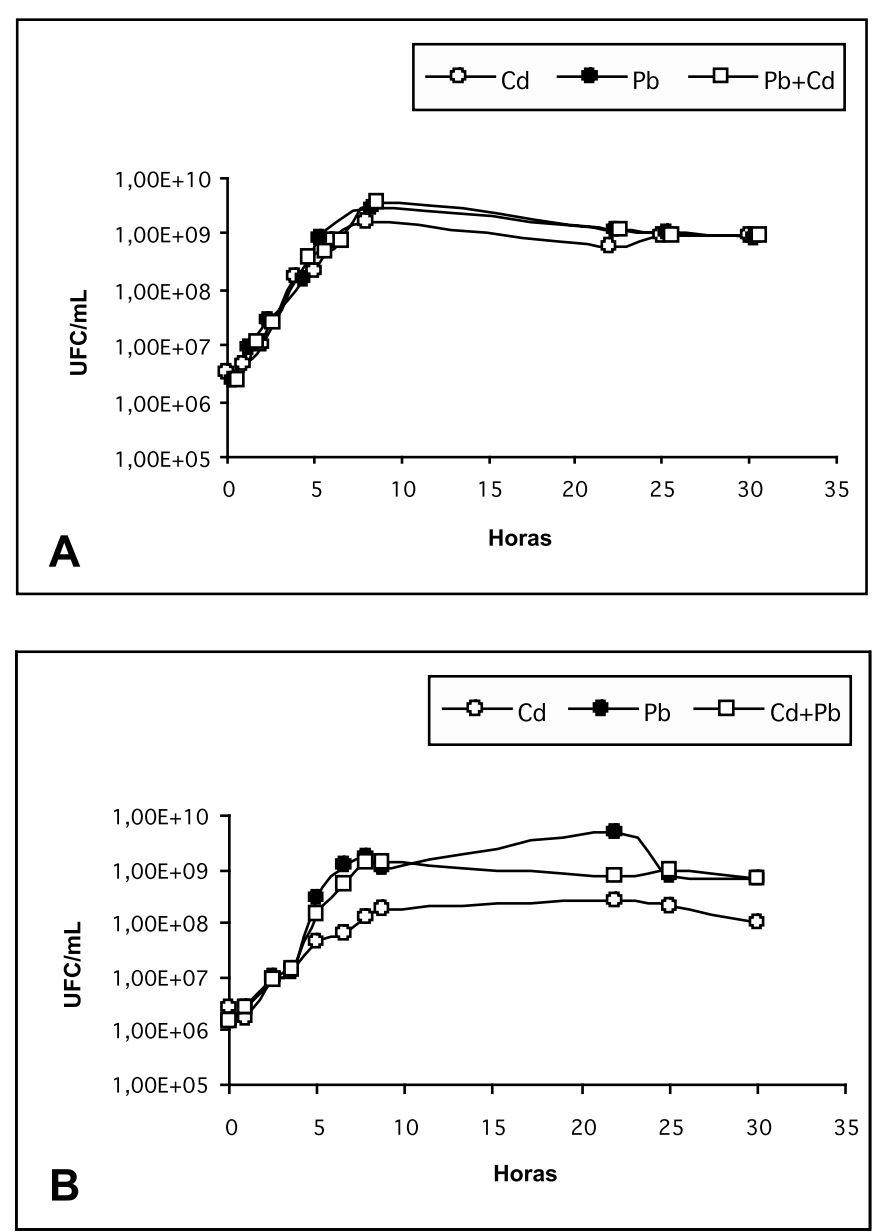

Figura 2. Curvas de crecimiento de E. coli en presencia de metales pesados. A: Crecimiento en presencia de una concentración de metales de $10 \mathrm{mg} / \mathrm{L}$. B: Crecimiento en presencia de una concentración de metales de $100 \mathrm{mg} / \mathrm{L}$.

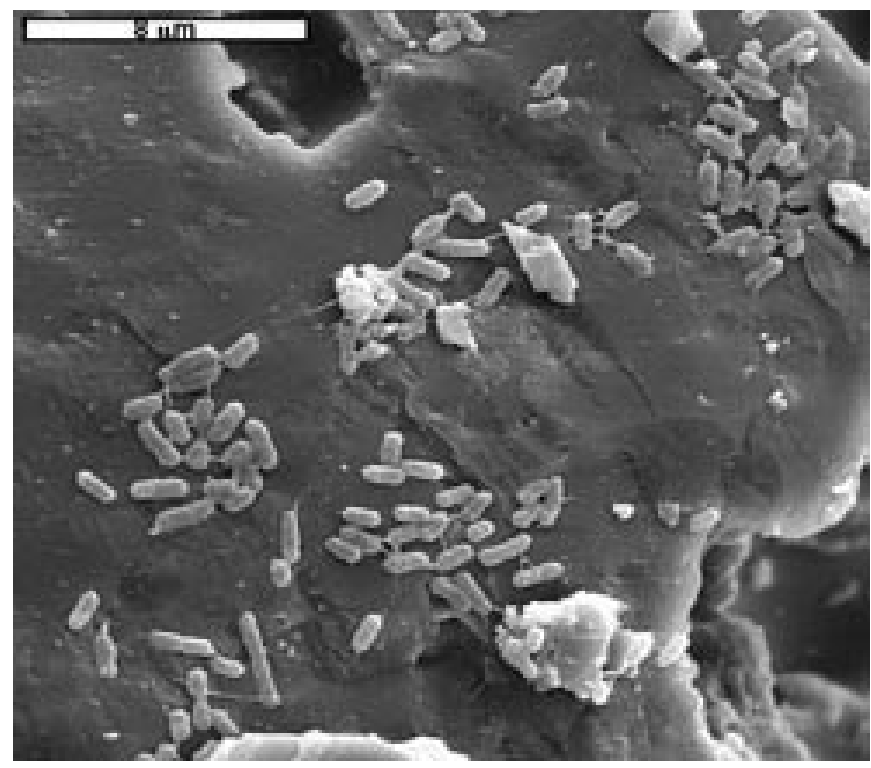

Figura 3. Cultivo de 24 horas de E. coli sobre material vitrocerámico. Micrografía de SEM. 
de metales por la acción microbiana a través de diferentes mecanismos como pueden ser la biosorción, bioacumulación, bioprecipitación, etc. Todos estos mecanismos se engloban dentro del término biorremediación, que en este caso particular implica el aprovechamiento de la capacidad microbiana para reducir y/o eliminar la toxicidad de los metales pesados de una solución contaminada mediante la reducción de su concentración en el medio. En estos procesos las sustancias poliméricas extracelulares (EPS) de la biopelícula desempeñan un papel muy importante, pues tienen multitud de sitios de unión para los metales pesados (15).

Los resultados de este trabajo suponen una contribución a los estudios de biorremediación de metales pesados de medios acuosos, poniendo de manifiesto la participación microbiana en la eliminación de los metales pesados mediante la formación de biopelículas y abren la posibilidad de utilizar el material vitrocerámico elaborado como soporte en un proceso de biorremediación de aguas contaminadas por metales pesados.

\section{AGRADECIMIENTOS}

Quisiéramos expresar nuestra gratitud a la Comisión Interministerial de Ciencia y Tecnología por su financiación (CICYT, proyectos AMB99-1187 y DP12000-0153-P4-0) y a las empresas Mahou S.A. y ESMALTES S.A por el suministro de parte de las materias primas y el seguimiento activo del proyecto.

\section{BIBLIOGRAFÍA}

1. M. Loaéc, R. Olier, J. Guezennec. "Uptake of lead, cadmium and zinc by a novel baterial exopolysacharide". Water Research. 31 (5), 1171-1179. (1997).

2. E. Salinas, M. Elorza de Orellano, I. Rezza, L. Martínez, E. Marchesvky,
M. Sanz de Tosetti. "Removal of cadmium and lead from dilute aqueous solutions by Rhodotorula rubra. Bioresource Technology". 72, 107-112. (2000).

3. H. Eccles. "Treatment of metal-contaminated wastes: why select a biological process?" Trends in Biotechnology. 17, 462-465. (1999).

4. G.M. Gadd. "Bioremedial potencial of microbial mechanisms of metal mobilization and Immovilization". Current Opinion in Biotechnology. 11, 271279. (2000).

5. M. Romero, J. Ma. Rincón. “Glass-Ceramics as building materials”. Mater. Construcc. 46, 91-106 y 242-243, (1996)

6. M. Romero, J. Ma. Rincón. "The controlled vitrification/crystallization process to the recycling of inorganic wastes". Bol. Soc. Esp. Ceram. V., 39 (1) 155-163 (2000).

7. K.S. TenHuisen, P.W Brown. "Phase evolution during the formation of tricalcium phosphate". Journal of the American Ceramic Society. 82 (10) 28132818. (1999).

8. M-P. Ginebra, E. Fernández, F.C.M. Driessens, J.A. Planell. “Modelling of the hydrolysis of tricalcium phosphate". Journal of the American Ceramic Society. 82 (10) 2808-2812. (1999).

9. J.M. Villora, P. Callejas, M. F. Barba. “Métodos de síntesis y comportamiento térmico del hidroxiapatito". Bol. Soc. Esp.Ceram.V., 41 (5) 443-450 (2002).

10. J. Llanos, C. Capasso, E. Parisi, D. Prieur, C. Jeanthon. "Susceptibility to heavy metals and cadmium accumulation in aerobic and anaerobic thermophilic microorganisms isolated from deep-sea hydrothermal vents". Current Microbiology. 41 (3), 201-205. (2000).

11. C. Geslin, J. Llanos, D. Prieur, C. Jeanthon. "The manganese anf iron superoxide dismutases protect Escherichia coli from heavy metal toxicity". Research Microbiology. 152 (10), 901-905. (2001).

12. R. Grimaud, B. Ezraty, J. K. Mitchell, D. Laffite, C. Briand, P.J. Derrick, F. Barras. "Repair of oxidized proteins. Identification of a new methionine sulfoxide reductase". Journal of Biological Chemistry, 276 (52), 48915-48920. (2001).

13. J.W. Costerton, Z. Lewandowski. "Microbial Biofilms". Annual Review Microbiology. 49, 711-745. (1995).

14. K.S. Low, C.K. Lee, S.C. Liew. "Sorption of cadmium and lead from aqueous solutions by spent grain". Process Biochemistry. 36, 59-64. (2000).

15. K. Friese, M. Mages, K. Wendt-Potthoff, T.R. Neu. "Determination of heavy metals in biofilms from the River Elbe by total-reflection X-ray fluorescence spectometry". Spectrochimica Acta. Part B. 52, 1019-1025. (1997).

Recibido: 01.02.03

Aceptado: 30.11 .03 